\title{
Towards the Intelligent Detection and Multimodal Rehabilitation for Cognitive Disabilities
}

\author{
Zengguang Hou
}

\begin{abstract}
The ageing of the population drives the rapid increase of cognitive disorders, which cause a heavy burden for families and nations. It is important to screen and interfere with cognitive disorders at the earlier stage, but we are short of affordable and effective approaches. In this talk, we will discuss our attempt in the design of the multi-mode detection and rehabilitation methods for cognitive disorders using computational intelligence algorithms, wearable devices and rehabilitation robots.
\end{abstract}

\section{Z. Hou $(\bowtie)$}

State Key Laboratory of Management and Control for Complex Systems (Key Laboratory of Complex Systems and Intelligence Science), Institute of Automation, Chinese Academy of Sciences, 95 Zhongguancun East Road, Beijing, Haidian District, China

e-mail: zengguang.hou@ia.ac.cn 